\title{
PLANT MITOCHONDRIA GROUP II INTRONS SPLICING: A WINDOW INTO THE EVOLUTION OF THE NUCLEAR SPLICEOSOMAL MACHINERIES
}

\author{
Oren Ostersetzer-Biran \\ Department of Plant and Environmental Sciences, \\ The Alexander Silberman Institute of Life Sciences, \\ The Hebrew University of Jerusalem, Givat-Ram, Jerusalem, Israel
}

Mitochondria serve as principal sites for cellular energy metabolism and play pivotal Mitochondria serve as principal sites for cellular energy metabolism and play pivotal roles in the biosynthesis of many essential metabolites for the (plant) cell. As descendants of a freeliving organism, mitochondria contain their own genome, the mtDNA. The mtDNAs in plants are notably larger and more complex in structure than their corresponding ones in Animalia. Plant mitochondria are also remarkable with respect to the presence of numerous group II introns that reside in many organellar genes. The removal of the introns from the coding sequences they interrupt is essential for respiratory functions and is mediated by enzymes that belong to a diverse set of protein-families. These include intron-encoded related proteins (i.e. maturases) that function in the splicing of group II introns in bacteria and mitochondria in fungi and plants, usually with high specificity towards the intron in which they are encoded. While the splicing of group II introns in vivo is facilitated by maturase factors, canonical group II introns are catalytic RNAs that are able to excise themselves from their pre-RNA hosts in vitro, in the absence of the protein cofactors, using a mechanism identical to that utilized by the spliceosome. Structural analyses and phylogenetic data may indicate that the spliceosomal RNAs have evolved from group II intron-related ancestors. Yet, it remains unclear how could such general players in spliceosomal splicing evolve from the monospecific bacterial systems (i.e. a group II intron RNAs and their highly specific intron-encoded maturase factors). Analysis of the organellar splicing machinery in plants may provide us with important clues into the evolution of the nuclear splicing machineries. Genetic and biochemical studies led to the identification of different protein factors that facilitate the splicing of many of the mitochondrial introns in plants. We established the native RNA targets of different maturase factors in plants and analyzed the organellar and developmental defects associated with their mutant lines in vivo. Interestingly, while model maturases in bacteria and fungi mitochondria act specifically on their cognate intron RNAs, the plant maturases are acting on multiple mtRNA targets, thus seem to be acting as organellar proto-spliceosomal factors. The ability of the mitochondrial maturases in plants to act on different intron targets further support the notion that the early organellar self-splicing and mobile group II RNAs spread in the eukaryotic genomes and later 'degenerated' into the universal splicing system, known as the spliceosome. The similarities between maturases and the core spliceosomal factor, Prp8, may support this intriguing hypothesis. 